University of New Hampshire

University of New Hampshire Scholars' Repository

Space Science Center

Institute for the Study of Earth, Oceans, and

Space (EOS)

2005

\title{
A hard X-ray polarimeter designed for transient astrophysical sources
}

Mark L. McConnell

University of New Hampshire - Main Campus, mark.mcconnell@unh.edu

Peter F. Bloser

University of New Hampshire, Peter.Bloser@unh.edu

Jason S. Legere

University of New Hampshire, jslegere@unh.edu

John R. Macri

University of New Hampshire - Main Campus, John.Macri@unh.edu

T Narita

College of the Holy Cross

Follow this and additional works at: https://scholars.unh.edu/ssc

Part of the Astrophysics and Astronomy Commons

\section{Recommended Citation}

McConnell, M.L.; Bloser, P.; Legere, J.; Macri, J.R.; Narita, T.; Ryan, J.M., "A hard X-ray polarimeter designed for transient astrophysical sources," Nuclear Science Symposium Conference Record, 2005 IEEE , vol.1, no., pp.462,466, 23-29 Oct. 2005

This Conference Proceeding is brought to you for free and open access by the Institute for the Study of Earth, Oceans, and Space (EOS) at University of New Hampshire Scholars' Repository. It has been accepted for inclusion in Space Science Center by an authorized administrator of University of New Hampshire Scholars' Repository. For more information, please contact Scholarly.Communication@unh.edu. 


\title{
A Hard X-Ray Polarimeter Designed for Transient Astrophysical Sources
}

\author{
M. L. McConnell, Member, IEEE, P. Bloser, J. Legere, J. R. Macri, T. Narita, J. M. Ryan
}

\begin{abstract}
This paper discusses the latest progress in the development of GRAPE (Gamma-Ray Polarimeter Experiment), a hard X-ray Compton Polarimeter. The purpose of GRAPE is to measure the polarization of hard $X$-rays in the $50-300 \mathrm{keV}$ energy range. We are particularly interested in $\mathrm{X}$-rays that are emitted from solar flares and gamma-ray bursts (GRBs). Accurately measuring the polarization of the emitted radiation from these sources will lead, to a better understating of both the emission mechanisms and source geometries. The GRAPE design consists of an array of plastic scintillators surrounding a central high-Z crystal scintillator. We can monitor individual Compton scatters that occur in the plastics and determine whether the photon is photo absorbed by the high-Z crystal or not. A Compton scattered photon that is immediately photo absorbed by the high- $Z$ crystal constitutes a valid event. These valid events provide us with the interaction locations of each incident photon and ultimately produces a modulation pattern for the Compton scattering of the polarized radiation. Comparing with Monte Carlo simulations of a $100 \%$ polarized beam, the level of polarization of the measured beam can then be determined. The complete array is mounted on a flat-panel multi-anode photomultiplier tube (MAPMT) that can measure the deposited energies resulting from the photon interactions. The design of the detector allows for a large field-ofview ( $>\pi$ steradian), at the same time offering the ability to be close-packed with multiple modules in order to reduce deadspace. We plan to present in this paper the latest laboratory results obtained from GRAPE using partially polarized radiation sources.
\end{abstract}

Index Terms - X-ray astronomy detectors, X-ray measurements, Gamma-ray astronomy detectors, Polarimetry, Photomultipliers, Position sensitive particle detectors, Data acquisition.

\section{INTRODUCTION}

$\mathbf{P}$ OLARIZATION measurements have become a powerful tool for astronomers throughout the electromagnetic spectrum. It is believed that by accurately measuring hard X-ray polarization levels from solar flares and gamma ray bursts (GRBs) we will be able to better understand both the emission mechanisms and source geometries producing the observed radiation. [1] With this goal in mind, we have been developing a hard X-ray polarimeter design that we call GRAPE (GammaRay Polarimeter Experiment). The purpose of GRAPE is to measure the polarization of hard x-rays in the $50-300 \mathrm{keV}$ energy range. As described here, the GRAPE design is most

Manuscript received January 20, 2002; revised August 13, 2002.

M. L. McConnell (telephone: 603-862-2047, e-mail: Mark.McConnell@unh.edu), P. Bloser, J. Legere, J. R. Macri, and J. M. Ryan are with the Space Science Center, University of New Hampshire, Durham, NH 03824 USA.

T. Narita is with the Department of Physics, College of the Holy Cross, Worcester, MA USA suitable for studies of either gamma-ray bursts or solar flares, as part of a long-duration balloon platform or as part of a satellite platform.

\section{COMPTON POLARIMETRY}

The basic physical process used to measure polarization in the 50-300 keV energy range is Compton scattering [1], the cross-section for which is given by [3],

$$
d \sigma=\frac{r_{0}^{2}}{2} d \Omega\left(\frac{\nu^{\prime}}{\nu_{o}}\right)^{2}\left(\frac{\nu_{o}}{\nu^{\prime}}+\frac{\nu^{\prime}}{\nu_{o}}-2 \sin ^{2} \theta \cos ^{2} \eta\right)
$$

where,

$$
\frac{\nu^{\prime}}{\nu_{o}}=\frac{1}{1+\left(\frac{h \nu_{o}}{m c^{2}}\right)(1-\cos \theta)}
$$

Here $\nu_{o}$ is the frequency of the incident photon, $\nu^{\prime}$ is the frequency of the scattered photon, $\theta$ is the scattering angle of the scattered photon measured from the direction of the incident photon, and $\eta$ is the azimuthal angle of the scattered photon measured from the plane containing the electric vector of the incident photon. For a given value of $\theta$, the scattering cross section for polarized radiation reaches a minimum at $\eta=0^{\circ}$ and a maximum at $\eta=90^{\circ}$. In other words, photons tend to be scattered at a right angle with respect to the incident electric field vector. In the case of an unpolarized beam of incident photons, there will be no net positive electric field vector and therefore no preferred azimuthal scattering angle $(\eta)$; the distribution of scattered photons with respect to $\eta$ will be uniform. However, in the polarized case, the incident photons will exhibit a net positive electric field vector and the distribution in $\eta$ will be asymmetric.

The ultimate goal of a Compton scatter polarimeter is to measure the azimuthal modulation pattern of the scattered photons. From Eq. 1, we see that the azimuthal modulation follows a $\cos 2 \eta$ distribution. More specifically, we can write the integrated azimuthal distribution of the scattered photons as,

$$
C(\eta)=A \cos \left(2\left(\eta-\phi+\frac{\pi}{2}\right)\right)+B
$$

where $\phi$ is the polarization angle of the incident photons. It is customary to characterize the azimuthal modulation in terms of the polarization modulation factor [1], [2]. For an incident beam at a given energy and incidence angle and with a polarization 


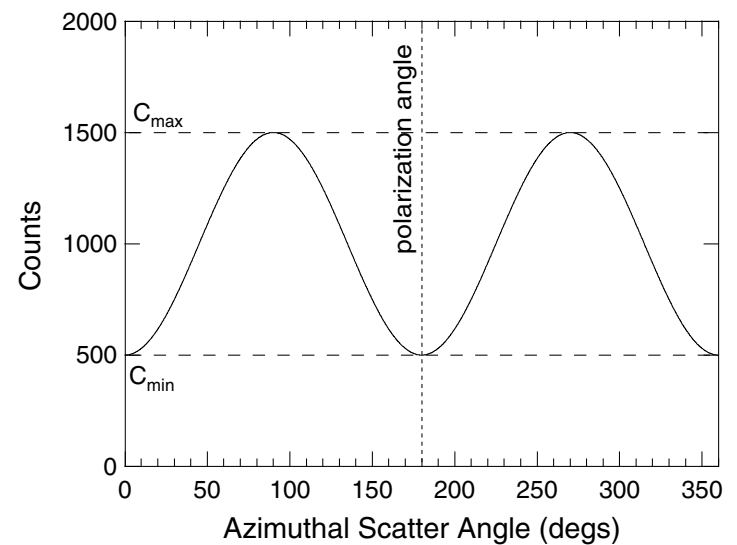

Fig. 1. The modulation pattern produced by Compton scattering of polarized radiation. The minimum of the modulation pattern defines the plane of polarization of the incident flux. In this case, $\phi=180^{\circ}$.

level denoted by $p$, the modulation factor $\left(\mu_{p}\right)$ can be expressed as,

$$
\mu_{p}=\frac{C_{p, \max }-C_{p, \min }}{C_{p, \max }+C_{p, \min }}=\frac{A_{p}}{B_{p}}
$$

where $C_{p, \max }$ and $C_{p, \min }$ refer to the maximum and minimum number of counts registered in the polarimeter, respectively, with respect to $\eta ; A_{p}$ and $B_{p}$ refer to the corresponding parameters in equation (3). We define a modulation factor for an incident beam that is $100 \%$ polarized $\left(\mu_{100}\right)$ based on simulations. This result is used, in conjunction with the observed modulation factor $\left(\mu_{p}\right)$, to determine the level of polarization $(P)$ in a measured beam,

$$
P=\frac{\mu_{p}}{\mu_{100}}
$$

The minimum detectable polarization (MDP) can be expressed as [2],

$$
\operatorname{MDP}(\%)=\frac{n_{\sigma}}{\mu_{100} S} \sqrt{\frac{2(S+B)}{T}}
$$

where $n_{\sigma}$ is the significance level (number of sigma), $S$ is the total source counting rate, $B$ is the total background counting rate and $T$ is the total observation time. Improved sensitivity to source polarization can be achieved either by increasing the modulation factor $\left(\mu_{100}\right)$ or by increasing the effective area of the polarimeter (thereby increasing the source counting rate, $S)$.

\section{THE GRAPE CONCEPT}

The development and design of the GRAPE detector has evolved through three science models. [4]-[12] Each one represented a successive improvement, but all three essentially operate under the same underlying principle of operation. A high- $\mathrm{Z}$ material, the calorimeter, is surrounded by multiple plastic scintillation detectors that serve as a target for the Compton scattering. The plastic scintillators are made of a low- $\mathrm{Z}$ material that maximizes the probability of a Compton interaction. The purpose of the calorimeter is to fully absorb the energy of the scattered photon. Ideally, photons that are incident on the plastic scintillator array will Compton scatter only once, and then be subsequently absorbed by the calorimeter. For such an event we measure the energy of the scattered electron in the plastic and the deposited energy of the scattered photon in the calorimeter. With multiple plastic scintillators surrounding the calorimeter, we can determine the azimuthal scatter angle of each valid event. A histogram of these data represents the azimuthal modulation pattern of the scattered photons, which provides a measurement of the polarization parameters (magnitude of the polarization and polarization angle) of the incident flux.

In order to accurately measure the azimuthal modulation (and hence the polarization parameters), we need to correct for geometric effects specific to the individual detector design. When the azimuthal modulation profile is generated, the distribution not only includes the intrinsic modulation pattern due to the Compton scattering process, but it also includes various geometric effects. One of these effects originates from the specific layout of the detector elements within the polarimeter and the associated quantization of possible scatter angles. Other effects include such things as the nonuniform detection efficiency of the PMT used for detector readout. In order to properly account for these effects, the response of the polarimeter must be properly characterized, for example, by measuring the response of the polarimeter to an unpolarized photon beam.

To determine the polarization level, $\mathrm{P}$, of the incident radiation we need to know the modulation factor for a completely polarized beam $\left(\mu_{100}\right)$. We have used simulations based on MGEANT (incorporating the GLEPS polarization code) to model the response of the polarimeter to $100 \%$ polarized incident radiation. The simulations included all important components of the lab setup.

\section{THE GRAPE DESIGN}

\section{A. Hardware}

The latest version of GRAPE (Science Model 3 - SM3) is a compact design based on the use of flat-panel MAPMT (Hamamatsu H8500). [11] The H8500 is a MAPMT with an array of $8 \times 8$ independent anodes. The $5 \mathrm{~mm}$ anodes are arranged with a pitch of $6 \mathrm{~mm}$, occupying a total area of 52 $\mathrm{mm} \times 52 \mathrm{~mm}$. The tube depth is only $28 \mathrm{~mm}$. Each GRAPE module includes a complete array of both plastic scattering elements and a $\operatorname{CsI}(\mathrm{Na})$ calorimeter element mounted on the front surface of the MAPMT (as seen in Fig. 2). The MAPMT provides readout of both scintillator types. The entire assembly is housed in a light-tight aluminum conatiner. The square design of the MAPMT also allows for the close packing of multiple modules with minimal dead space.

Preamp boards were designed to be attached to the output pin assembly on the base of the MAPMT (Fig. 2). Each of the four preamp boards handles 16 anode channels plus 1 test channel. All signals are subsequently processed using NIM spectroscopy 


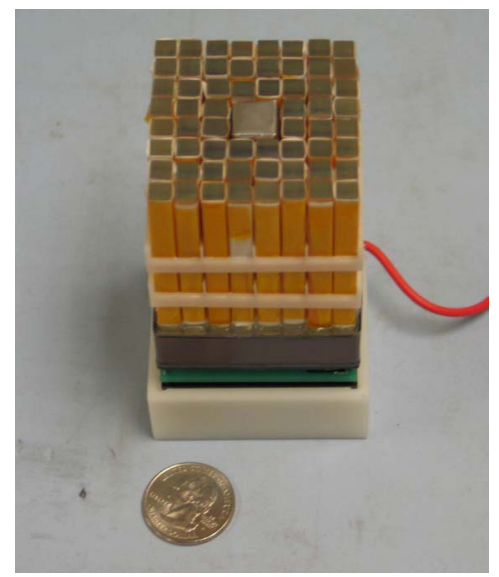

Fig. 2. GRAPE Science Model 3 (SM3) fully populated with 60 plastic scintillators and a central $\mathrm{CsI}(\mathrm{Na})$ calorimeter.

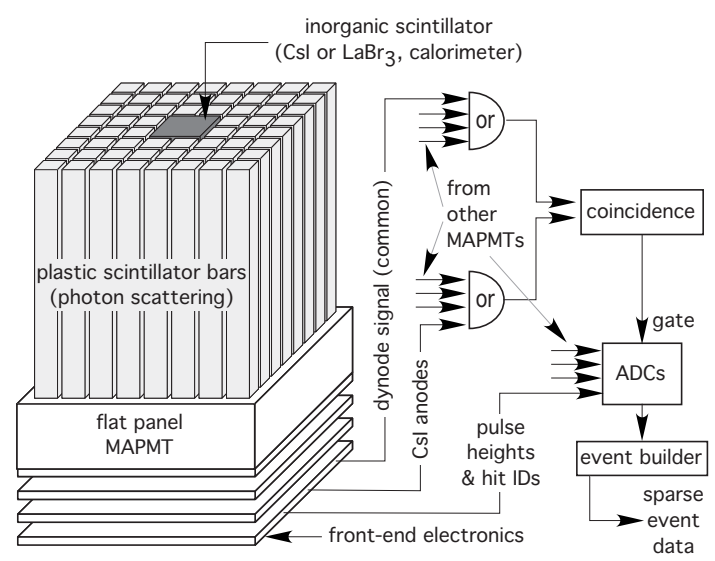

Fig. 3. GRAPE logic diagram, illustrating the signal processing involved.

amplifiers (CAEN model N568), which provide both a fast and a slow output. The fast outputs are used for timing purposes and the slow outputs are used for pulse height analysis.

Signal processing is controlled, in part, by a VME-based constant fraction discriminator (CFD, CAEN model V812) with 16-channel capability. In order to use the 16-channel CFD to monitor the 60 fast signals from the plastic scintillators, a passive sum box was designed and built to combine the signals from the plastic scintillators into 15 groups. The gain-matched fast signals from each group were sent to the sum box and each sum output sent to the CFD. The CFD has an OR output option, which functions by outputting a logic signal when one of its 16-channels is above the programmed threshold. This works because we are interested in single events occurring in any one plastic. When a signal above the CFD threshold occurs in any one plastic group, a logic signal is sent to the logic portion of the setup for coincidence determination.

The VME-based CFD only monitors the fast plastic signals. Separate NIM-based CFDs are used for the polarizer and calorimeter channel. The NIM-based CFDs are adjusted individually for threshold and delays. Each CFD will then send

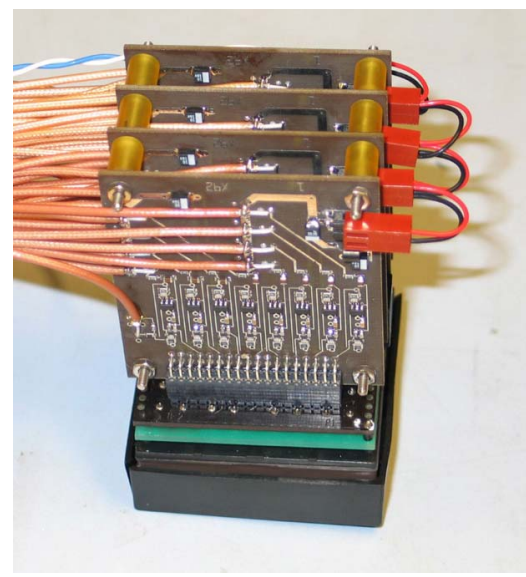

Fig. 4. The preamp circuit boards plugged into the back end of the MAPMT.

a logic signal to the coincidence portion of the setup when programmable threshold levels are exceeded for each detector.

The coincidence (logic) piece of the setup consists of 1) a PLS 794 Quad gate generator 2) a LeCroy 365AL 4-Fold Logic Unit 3) a LeCroy 222 Dual gate generator. The three logic signals (plastic, CsI(NaI), polarizer) are input to the PLS 794 in order to create gates for each channel. The gate widths are set according to the speed of each detector, the CsI $(\mathrm{NaI})$ having a much slower rise time compared to the plastics. These gates are fed to the logic unit where an AND circuit allows the user to select the level of coincidence. For example, a polarized run would be set to have the three gates active, requiring a triple coincidence level. When energy calibrations are needed, the appropriate detector can be selected and the level of coincidence set to single.

The output of the logic unit generates a gate (LeCroy model 222 Dual Gate Generator) that is used to trigger ADC conversion. Each slow signal from the MAPMT is sent to VMEbased ADCs (CAEN model M785) for pulse height conversion.

Controlling the operation of the VME crate, the two ADC modules and the CFD module is achieved using KMAX software version 8.03 (from Sparrow Corp.) running on a Macintosh G4 computer (Mac OS X 10.3). KMAX communicates to VME modules through a fiber optic cable connected from an SBS 620 controller VME card to an SBS PCI card in the Mac. The data are written to event files that are subsequently analyzed using the ROOT software package.

\section{B. Laboratory Measurements}

Experiments were conducted in the laboratory in order to determine the effectiveness of the detector. The polarized radiation used in the tests was produced by Compton scattering radiation from a laboratory gamma-ray source. [5] Radiation from a ${ }^{137} \mathrm{Cs}$ source is collimated with lead shielding and directed toward a plastic scintillation detector, referred to as the polarizer. Photons with an initial energy of $662 \mathrm{keV}$ scatter through an angle of $90^{\circ}$ before reaching the polarimeter detector. The radiation reaching the polarimeter is highly polarized 

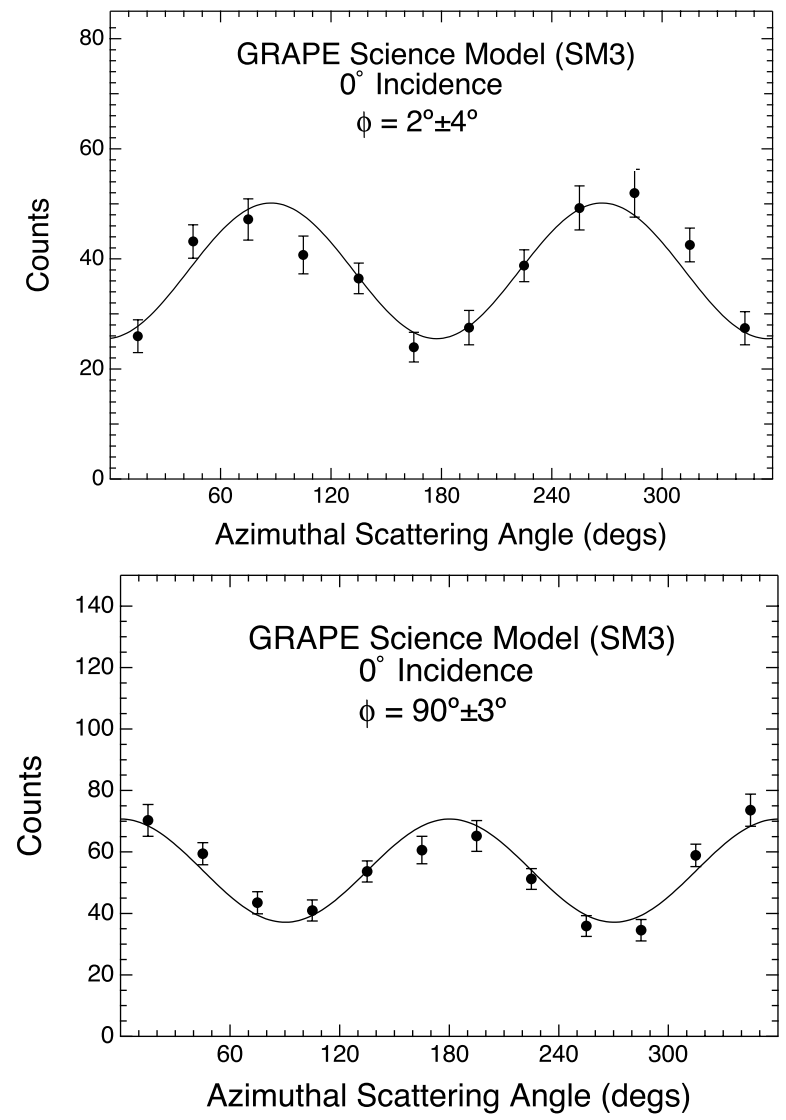

Fig. 5. Results from SM3 with the inner plastic ring eliminated from the analysis/

(at a level of 55-60\%) and has a reduced energy of $288 \mathrm{keV}$. The use of the polarizer also allows us to electronically tag photons. A triple coincidence between the polarizer and the two sets of polarimeter detectors provides an efficient means for recording data from the polarized beam.

Initial laboratory tests of SM3 proved unsuccessful. It was discovered that there were light cross-talk issues between the calorimeter and the twelve plastic elements immediately surrounding the calorimeter (c.f., Figure 2). We believe that this can be attributed to the close proximity between the calorimeter and adjacent plastics. With a light output of $\sim 4$ times that of a plastic scintillator, the CsI(NaI) calorimeter was contributing to the energy recorded in this inner plastic ring. We are currently investigating various options for mitigating this cross talk problem. In the meantime, in order to properly analyze the SM3 data, we decided to eliminate the inner twelve plastic scintillator elements from the analysis. This left 48 plastic elements in the array for inclusion in the data analysis. The data shown in Fig. 5 represent the results of an analysis that excluded the innermost plastic elements. Data was collected for $0^{\circ}$ and $90^{\circ}$ polarization angle and yielded polarization levels of $56( \pm 9) \%$ and $55( \pm 7) \%$ respectively.

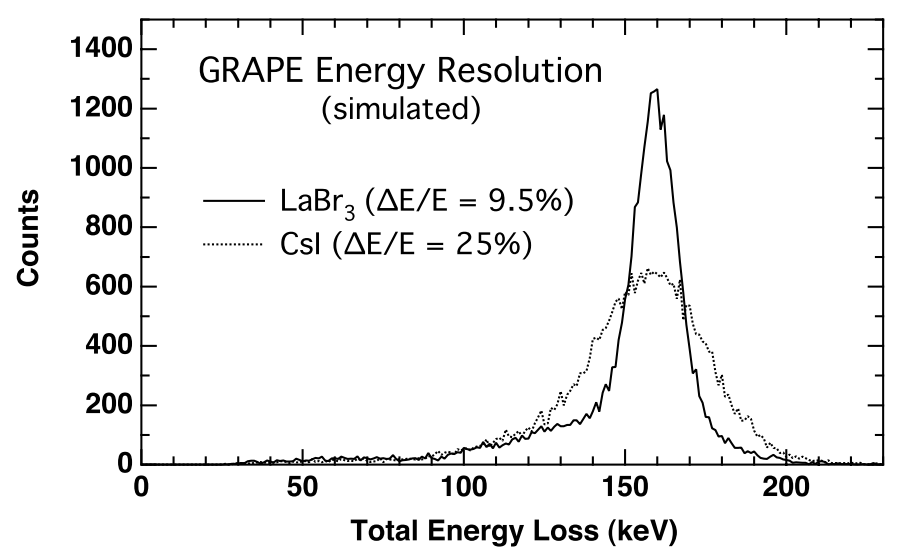

Fig. 6. Simulated results showing the improved energy resolution of the SM4 design that would result from changing the CsI calorimeter to one made from $\mathrm{LaBr}_{3}$.

\section{GRAPE FUTURE}

The next major step for GRAPE development will be to improve the coincidence timing and energy resolution. We plan to accomplish this by replacing the central CsI calorimeter with one based on Lanthanum Bromide $\left(\mathrm{LaBr}_{3}\right)$. [13], [14] This relatively new inorganic scintillator provides an energy resolution that is more than twice as good as $\mathrm{NaI}$ at $662 \mathrm{keV}$ (3\% vs. $7 \%$ ). The expected improvement in the GRAPE energy resolution (combining the energy resolution of both the plastic scattering elements and the calorimeter) is shown in Fig. 6. The $\mathrm{LaBr}_{3}$ will also provides for better timing characteristics. With decay times of $\sim 25 \mathrm{nsec}$, it is comparable to the plastic scintillator and will greatly improve the coincidence timing characteristics of GRAPE.

In order to provide adequate sensitivity, any realistic application of the GRAPE design would involve an array of polarimeter modules. One possible deployment option for a GRAPE polarimeter array would be as the primary instrument on an Ultra-Long Duration Balloon (ULDB) payload. The ULDB technology currently under development by NASA is expected to provide balloon flight durations of up to 100 days. A $1 \mathrm{~m}^{2}$ array of GRAPE modules would easily fit within the envelope of a ULDB payload. The ideal configuration for GRB studies would be an array that remains pointed in the vertical direction (i.e., towards the zenith) at all times. In this case, there would be no pointing requirements, only a moderate level of aspect information (continuous knowledge of the azimuthal orientation to $\sim 0.5^{\circ}$ ). An imaging polarimeter could also be designed to match the payload limitations of a ULDB, although the pointing requirements would be much more severe $\left(<1^{\circ}\right.$ in both azimuth and zenith). A second deployment option would be as part of a spacecraft payload.

\section{ACKNOWLEDGMENT}

This work is currently supported by NASA grants NNG04GB83G and NNG04WC16G. We would like to thank Mark Widholm and Paul Vachon for their support with the 


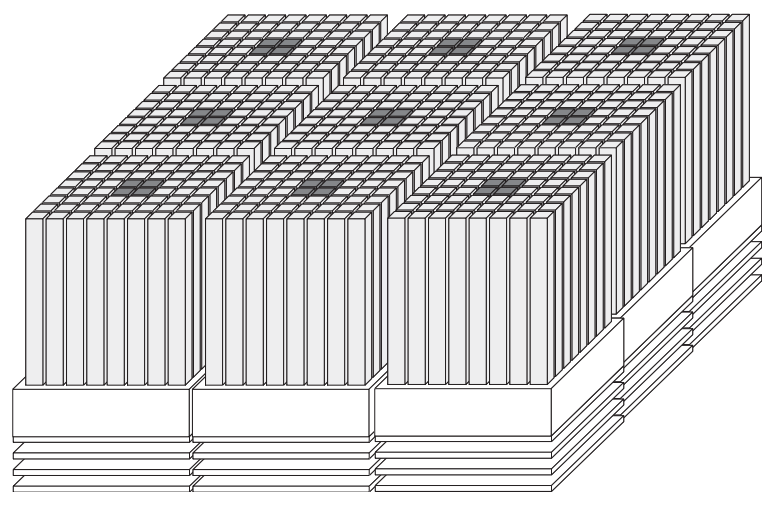

Fig. 7. An array of nine GRAPE modules.

MAPMT design electronics. We would also like to thank the Laboratory for Advanced Instrumentation Research at Embry Riddle, Sparrow Corp., Drew Weisenberger, and Sergio Brambilla for their support with the VME data acquisition setup.

\section{REFERENCES}

[1] F. Lei, A.J. Dean and G.L. Hills, "Compton scatter polarimetry in gammaray astronomy," Space Sci. Rev. 82, pp. 309-388, 1997.

[2] R. Novick, "Stellar and solar X-ray polarimetry," Space Sci. Rev. 18, pp. 389-408, 1975.

[3] R. D. Evans, The Atomic Nucleus, McGraw-Hill, New York, 1958.

[4] M.L. McConnell, D.J. Forrest, J. Macri, J.M. Ryan, and W.T. Vestrand, "Development of a hard X-ray polarimeter for gamma-ray bursts," in AIP Conf. Proc. 428, Gamma-Ray Bursts, C.A. Meegan and P. Cushman, ed., pp. 889-893, AIP, New York, 1998.

[5] M.L. McConnell, D.J. Forrest, J. Macri, M. McClish, M. Osgood, J.M. Ryan, W.T. Vestrand and C. Zanes "Development of a hard X-ray polarimeter for solar flares and gamma-ray bursts," IEEE Trans. Nucl. Sci. 45, pp. 910-914, 1998.

[6] M.L. McConnell, J.R. Macri, M. McClish, J. Ryan, D.J. Forrest and W.T. Vestrand, "Development of a hard X-ray polarimeter for astrophysics," IEEE Trans. Nucl. Sci 46, pp. 890, 1999.

[7] M.L. McConnell, J.R. Macri, M. McClish, and J. Ryan, "Recent laboratory tests of a hard X-ray solar flare polarimeter," SPIE Proc. 3764, pp. 70-78, 1999.

[8] M. L. McConnell, J. R. Macri, and J. M. Ryan, "A modular hard X-ray polarimeter for solar flares," in ASP Conf. Ser. 206, High Energy Solar Physics - Anticipating HESSI, R. Ramaty and N. Mandzhavidze, ed., pp. 280-283, ASP, San Francisco, 2000.

[9] M. L. McConnell, J. R. Ledoux, J. R. Macri, and J. M. Ryan, "A Hard X-ray Polarimeter for Gamma-Ray Bursts and Solar Flares," SPIE Proc. 4851, pp. 1382-1393, 2002.

[10] M. L. McConnell, J. R. Ledoux, J. R. Macri, and J. M. Ryan, "The Development of GRAPE, A Gamma-Ray Polarimeter Experiment," in AIP Conf. Proc. 662, Gamma-Ray Burst and Afterglow Astronomy, G. R. Ricker and R. K. Vanderspek, ed., pp.503-505, AIP, New York, 2003.

[11] M. L. McConnell, J. R. Ledoux, J. R. Macri, and J. M. Ryan, "Dedicated Polarimeter Design for Hard X-Ray and Soft Gamma-Ray Astronomy," SPIE Proc. 5165, pp. 334-345, 2004.

[12] J. S. Legere, P. Bloser, J. R. Macri, M. L. McConnell, T. Narita and J. M. Ryan, "Developing a Compton Polarimeter to Measure Polarization of Hard X-Rays in the 50-300 keV Energy Range," SPIE Proc. 5898, pp. 401-410, 2005.

[13] van Loef, E. V. D., et al., "High Energy Resolution Scintillator: $\mathrm{Ce}^{3+}$ Activated $\mathrm{LaBr}_{3}$, , Appl. Phys. Lett., 79, pp. 1573-1575, 2001.

[14] Shah, K. S., et al., " $\mathrm{LaBr}_{3}$ :Ce Scintillators for Gamma Ray Spectroscopy," IEEE Trans. Nucl. Sci., 50, pp. 2410-2413, 2003. 